\title{
Digital Learning Resources
}

No.

Portal

Address

Concern

1. e-resources Perpusnas

http://e-resources.perpusnas.go.id/

All fields

2. Rama Kemdikbud

https://rama.kemdikbud.go.id/

All fields

3. Anjani Kemdikbud

http://anjani.kemdikbud.go.id/

All fields

4. Garuda Kemdikbud

https://garuda.kemdikbud.go.id/

All fields

5. One search

https://onesearch.id

All fields

6. Neliti

https://www.neliti.com/id/

All fields

7. Moraref

https://moraref.kemenag.go.id

All fields

8. App Dimension

https://app.dimensions.ai/discover/publication

All fields

9. Perpustakaan Kemdikbud RI

https://perpustakaan.kemdikbud.go.id/

Education

10. Belajar Kemdikbud

https://belajar.kemdikbud.go.id

Education

11. Repository Kemdikbud

http://repositori.kemdikbud.go.id/

All fields

12. Summon Ristekdikti https://ristekdikti.summon.serialssolutions.com/

All fields

13. Google Scholar https://scholar.google.co.id/

All fields

14. Researchgate

https://www.researchgate.net/

All fields

15. Academia https://www.academia.edu/

All fields

16. Issuu

https://issuu.com/

All fields

17. PDF Drive

https://www.pdfdrive.com/

All fields

18. OSF Preprints

https://osf.io

All fields

19. RINarxiv (lipi.go.id)

https://rinarxiv.lipi.go.id/lipi

All fields

20. Indiarxiv

https://indiarxiv.org/

All fields

Natural Sciences,

Engineering,

21. ArXiv

https://arxiv.org

Economics,

Finance and

Computing

22. AgriXiv

https://agrixiv.org/

Agricultural

23. Arabixiv

https://arabixiv.org/

All fields

24. Biohackrxiv

https://biohackrxiv.org/

Biology, science

25. Bodoarxiv

https://osf.io/preprints/bodoarxiv

All fields

26. Earthxiv

https://eartharxiv.org/

Earth, Geography

27. Ecoevorxiv

https://ecoevorxiv.org/

28. Ecsarxiv

https://ecsarxiv.org/

Ecology, Science

29. Edarxiv

https://edarxiv.org/

All fields

All fields

30. Engrxiv

https://engrxiv.org/

Engineering,

31. Focus Archive

https://osf.io/preprints/focusarchive

All fields

32. Frencxiv

https://frenxiv.org/

All fields

33. Lawarxiv

https://osf.io/preprints/lawarxiv

Law,

34. Lissa

https://osf.io/preprints/lissa

All fields

35. Mediarxiv

https://mediarxiv.org/

All fields

36. Marxiv

https://marxiv.org/

All fields

ahmad.pratikno@trunojoyo.ac.id 


\begin{tabular}{|c|c|c|c|}
\hline 37. & Metaarxiv & https://osf.io/preprints/metaarxiv & All fields \\
\hline 38. & Mindrxiv & https://mindrxiv.org/ & All fields \\
\hline 39. & Nutrixiv & https://osf.io/preprints/nutrixiv & Nutrition, Science \\
\hline 40. & Paleorxiv & https://paleorxiv.org/ & $\begin{array}{l}\text { Paleontology, } \\
\text { Paleography }\end{array}$ \\
\hline 41. & Psyarxiv & https://psyarxiv.com/ & Psychology \\
\hline 42. & Socarxiv & https://osf.io/preprints/socarxiv & Social \\
\hline 43. & Sportxiv & https://osf.io/preprints/sportrxiv & Sport \\
\hline 44. & Thesis Commons & https://thesiscommons.org/ & All fields \\
\hline 45. & AfricArxiv & https://osf.io/preprints/africarxiv & All fields \\
\hline 46. & ChemRxiv & http://www.chemrxiv.org & Chemical Sciences \\
\hline 47. & SocArXiv & https://osf.io/preprints/socarxiv/ & Social Sciences \\
\hline 48. & ESSOAr & https://www.essoar.org & Earth Sciences \\
\hline 49. & $\begin{array}{l}\text { Research } \\
\text { Papers in Economics } \\
\text { (RePEc) }\end{array}$ & https://ideas.repec.org & Economics \\
\hline 50. & $\begin{array}{l}\text { Social Sciences Research } \\
\text { Network (SSRN) }\end{array}$ & https://www.ssrn.com/en & Social Sciences \\
\hline 51. & E-LIS & http://eprints.rclis.org & $\begin{array}{l}\text { Library and } \\
\text { Information } \\
\text { Science }\end{array}$ \\
\hline 52. & MDPI Preprints & https://www.preprints.org & $\begin{array}{l}\text { Natural, } \\
\text { Engineering, Social } \\
\text { Sciences and Arts } \\
\text { and Humanities }\end{array}$ \\
\hline 53. & PeerJ Preprints & https://peerj.com/preprints & $\begin{array}{l}\text { Biological, Medical, } \\
\text { Environmental and } \\
\text { Computing } \\
\text { Sciences }\end{array}$ \\
\hline 54. & bioRxiv & http://www.biorxiv.org & Life Sciences \\
\hline 55. & Archive & https://archive.org/ & All fields \\
\hline 56. & Preprints & https://preprints.org & All fields \\
\hline 57. & $\begin{array}{l}\text { BASE (Bielefeld Academic } \\
\text { Search Engine) }\end{array}$ & https://www.base-search.net & All fields \\
\hline 58. & Repository UT & http://repository.ut.ac.id/ & All fields \\
\hline 59. & $\begin{array}{l}\text { Repository Telkom } \\
\text { University }\end{array}$ & https://repository.telkomuniversity.ac.id & All fields \\
\hline 60. & Repository UNAIR & http://repository.unair.ac.id/ & All fields \\
\hline 61. & Repository ITS & $\begin{array}{l}\text { http://repository.its.ac.id/； } \\
\text { https://digilib.its.ac.id }\end{array}$ & All fields \\
\hline 62. & Repository ITB & https://repository.itb.ac.id & All fields \\
\hline 63. & Repository UNEJ & http://repository.unej.ac.id/ & All fields \\
\hline 64. & Repository UI & http://repository.ui.ac.id/ ; https://lib.ui.ac.id & All fields \\
\hline 65. & Repository UGM & http://repository.ugm.ac.id/ & All fields \\
\hline 66. & Repository UB & $\begin{array}{l}\text { http://repository.ub.ac.id/ ; } \\
\underline{\text { https://digilib.ub.ac.id }}\end{array}$ & All fields \\
\hline 67. & Repository UPI & http://repository.upi.edu/ & All fields \\
\hline
\end{tabular}




\begin{tabular}{|c|c|c|c|}
\hline 68. & $\begin{array}{l}\text { Repository IAIN } \\
\text { Tulungagung }\end{array}$ & http://repo.iain-tulungagung.ac.id/ & All fields \\
\hline 69. & $\begin{array}{l}\text { Repository UIN Syarif } \\
\text { Jakarta }\end{array}$ & http://repository.uinjkt.ac.id/ & All fields \\
\hline 70. & $\begin{array}{l}\text { Repository UIN Sunan } \\
\text { Kalijaga }\end{array}$ & http://lib.uin-suka.ac.id/\#digital & All fields \\
\hline 71. & Repository UIN Suska Riau & http://repository.uin-suska.ac.id/ & All fields \\
\hline 72. & $\begin{array}{l}\text { Repository Georgetown } \\
\text { University }\end{array}$ & https://repository.library.georgetown.edu/ & Islamic studies \\
\hline 73. & eThesis UIN Malang & http://etheses.uin-malang.ac.id/ & All fields \\
\hline 74. & Repository Univ. Gontor & http://repo.unida.gontor.ac.id/ & All fields \\
\hline 75. & Repository Univ. Nurul Jadid & http://repository.unuja.ac.id/ & All fields \\
\hline 76. & Repository Univ. Ibrahimy & https://libcat.ibrahimy.web.id & All fields \\
\hline 77. & Repository IAIN Salatiga & http://e-repository.perpus.iainsalatiga.ac.id/ & All fields \\
\hline 78. & Repository IAIN Kudus & http://perpustakaan.stainkudus.ac.id/ & All fields \\
\hline 79. & ePrints UIN Walisongo & http://eprints.walisongo.ac.id/ & All fields \\
\hline 80. & ePrints UAD & https://eprints.uad.ac.id & All fields \\
\hline 81. & ePrints UNY & https://eprints.uny.ac.id & All fields \\
\hline 82. & ePrints UNS & https://eprints.uns.ac.id & All fields \\
\hline 83. & ePrints UNDIP & https://eprints.undip.ac.id & All fields \\
\hline 84. & ePrints IAIN Surakarta & https://eprints.iain-surakarta.ac.id & All fields \\
\hline 85. & Library UNNES & $\underline{\text { https://lib.unnes.ac.id }}$ & All fields \\
\hline 86. & ePrints UTM & https://eprints.utm.my & All fields \\
\hline 87. & ePrints UPSI Malaysia & https://ir.upsi.edu.my & All fields \\
\hline 88. & ePrints Univ. Malaya & https://eprints.um.edu.my & All fields \\
\hline 89. & $\begin{array}{l}\text { Repository Univ. Islam } \\
\text { Sultan Syarif Ali, Brunei }\end{array}$ & $\begin{array}{l}\text { http://e- } \\
\text { ilami.unissa.edu.bn:8080/jspui/handle/1234567 } \\
\underline{89 / 10}\end{array}$ & All fields \\
\hline 90. & $\begin{array}{l}\text { Library Univ. Brunei } \\
\text { Darussalam (UBD) }\end{array}$ & http://library.ubd.edu.bn/Pages/default.aspx & All fields \\
\hline 91. & Digilib UM Malang & $\begin{array}{l}\text { https://digilib.um.ac.id ; } \\
\text { https://mulok.library.um.ac.id } \\
\text { http://repository.uin-malang.ac.id/ }\end{array}$ & All fields \\
\hline 92. & Digilib ITB Bandung & https://digilib.itb.ac.id & All fields \\
\hline 93. & Digilib UNESA & https://digilib.unesa.ac.id & All fields \\
\hline 94. & Digilib UMK Kudus & https://digilib.umk.ac.id & All fields \\
\hline 95. & Digilib UIN Sunan Ampel & $\begin{array}{l}\text { https://digilib.uinsby.ac.id ; } \\
\text { http://library.uinsby.ac.id/?page id=106 }\end{array}$ & All fields \\
\hline 96. & Digilib LIPI Bogor & https://digilib.biologi.lipi.go.id & Biology, science \\
\hline 97. & Digilib USM & https://digilib.usm.ac.id & All fields \\
\hline 98. & $\begin{array}{l}\text { Digilib UIN Sunan Gunung } \\
\text { Djati }\end{array}$ & https://digilib.uinsgd.ac.id/ & All fields \\
\hline 99. & Jogja Library & http://jogjalib.com/ & All fields \\
\hline 100. & $\begin{array}{l}\text { Open Access Thesis and } \\
\text { Dissertation }\end{array}$ & https://oatd.org/ & All fields \\
\hline 101. & $\begin{array}{l}\text { National Library of } \\
\text { Singapore }\end{array}$ & $\begin{array}{l}\text { http://eresources.nlb.gov.sg/arts/website/ } \\
\text { Common/Homepage.aspx }\end{array}$ & All fields \\
\hline
\end{tabular}


102. National Library of Malaysia http://myrepositori.pnm.gov.my/

All fields

103. National Library of Unite Kingdom

https://www.bl.uk/catalogues-and-

collections/digital-collections

All fields

104. National Library of

Netherland

http://www.digitalpreservation.gov/series/

edge/koninklijke.html

All fields

https://pro.europeana.eu/organisation/german-

105. National Library of Germany digital-library

http://www.libdex.com/country/germany

All fields

/berlin/library 22671.html

106. National Library of USA

https://www.loc.gov/collections/;

https://guides.library.harvard.edu/archives/us

All fields

107. National Library of Australia

https://www.nla.gov.au/content/national-

library-of-australia-digital-object-repository

All fields

108. National Library of Japan

https://www.nii.ac.jp/irp/en/list/

All fields

109. National Library of South Korea

http://www.nl.go.kr/english/

All fields

110. National Library of China National Library of

111. Hongkong http://www.nlc.cn/ All fields

https://www.hkpl.gov.hk/en/index.html

All fields

http://www.varastokirjasto.fi/en/;

112. National Library of Finland

https://www.kansalliskirjasto.fi/en/services/syst

All fields em-platform-services/repository-services

http://www.theeuropeanlibrary.org/tel4/contrib utor/P01224

https://www.regjeringen.no/en/dep/kud/

organisation/subordinate-agencies-and-related-

113. National Library of Norway

institutions-under-the-ministry-of-

All fields

culture/underliggende-etater/the-national-

library/id408537/

https://www.nb.no/en/digitizing-at-the-nationallibrary/

114. National Library of Canada http://www.bac-lac.gc.ca/eng/Pages/home.aspx

All fields

115. National Library of Egypt https://www.wdl.org/en/search

All fields

116. National Library of Saudi Arabia

https://kfnl.gov.sa/En/Pages/default.aspx

All fields

https://www.library.uaeu.ac.ae/en/

117. National Library of UAE https://tcaabudhabi.ae/en/what.we.do/national.l

All fields ibrary.aspx

118. National Library of Jordania https://library.ju.edu.jo/ http://www.nl.gov.jo/En/NL.aspx

All fields

119. National Library of Russia http://nlr.ru/eng/opac/

All fields

120. National Library of Poland

https://www.bn.org.pl/en

All fields

121. National Library of Sweden

http://www.sverigesdepabibliotekochlanecentral. se/english/

All fields

122. National Library of France https://www.bnf.fr/en/gallica-bnf-digital-library All fields https://eudocs.lib.byu.edu/index.php/Italy:

123. National Library of Italy Historical Collections

All fields

124. National Library of Spain http://www.bne.es/en/Inicio/index.html

All fields

125. National Library of Greece https://www.nlg.gr/

All fields

126. National Library of Turkey

http://www.mkutup.gov.tr/en/Sayfalar/default.a $\underline{\operatorname{spx}}$

All fields 


\begin{tabular}{|c|c|c|c|}
\hline 127. & Sciencedirect & https://www.sciencedirect.com/ & All fields \\
\hline 128. & Proquest & https://www.proquest.com/ & All fields \\
\hline 129. & ERIC & https://eric.ed.gov/ & All fields \\
\hline 130. & Sage Publishing & https://journals.sagepub.com/ & All fields \\
\hline 131. & Emerald & https://www.emerald.com/insight/ & All fields \\
\hline 132. & Taylor \& Francis & https://www.tandfonline.com/ & All fields \\
\hline 133. & Cambridge Core & $\begin{array}{l}\text { https://www.cambridge.org/core/what-we- } \\
\text { publish/journals }\end{array}$ & All fields \\
\hline 134. & IEEE & $\begin{array}{l}\text { http://ieeexplore.ieee.org/xpl/RecentIssue.jsp?pu } \\
\text { number=13 }\end{array}$ & All fields \\
\hline 135. & DOAJ & https://doaj.org/search & All fields \\
\hline 136. & JSTOR & http://www.jstor.org/ & All fields \\
\hline 137. & Hindawi & https://www.hindawi.com/journals/ & All fields \\
\hline 138. & Semanticscholar & https://www.semanticscholar.org/ & All fields \\
\hline 139. & Nadia Pub & https://nadiapub.com/ & All fields \\
\hline 140. & Plos One & https://journals.plos.org/plosone/ & All fields \\
\hline 141. & Plos One Preprints & https://plos.org/open-science/preprints/ & All fields \\
\hline 142. & Pubmed & http://www.ncbi.nlm.nih.gov/pubmed/ & All fields \\
\hline 143. & University of Oxford & $\begin{array}{l}\text { https://academic.oup.com/journals/pages/journ } \\
\text { als a to z; } \\
\text { https://academic.oup.com/DATABASE/ }\end{array}$ & All fields \\
\hline 144. & Wiley \& Blackwell & http://onlinelibrary.wiley.com/ & All fields \\
\hline 145. & Springer & https://link.springer.com/ & All fields \\
\hline 146. & Microsoft Academic & https://academic.microsoft.com/ & All fields \\
\hline 147. & Oxford University & $\begin{array}{l}\text { https://global.oup.com/academic/?cc=us\&lang=e } \\
\underline{\text { n\& }}\end{array}$ & All fields \\
\hline 148. & Brill & http://www.brill.com/search & All fields \\
\hline 149. & Ebsco & $\begin{array}{l}\text { https://www.ebsco.com/products/research- } \\
\text { databases/e-journals-database }\end{array}$ & All fields \\
\hline 150. & MDPI & https://www.mdpi.com/ & All fields \\
\hline 151. & Mc Grill & https://www.mheducation.com/ & All fields \\
\hline 152. & IGI Global & $\begin{array}{l}\text { https://www/igi- } \\
\text { global.com/journal/international-journal-web- } \\
\text { portals/1113 }\end{array}$ & All fields \\
\hline 153. & Perpustakaan Islam Digital & $\begin{array}{l}\text { https://perpustakaanislamdigital.com/index.php/ } \\
\text { fp/ }\end{array}$ & Islamic studies \\
\hline 154. & $\begin{array}{l}\text { Middle East and Islamic } \\
\text { Studies }\end{array}$ & https://brill.com/subject/HMEIS & Islamic studies \\
\hline 155. & Sciendo & https://www.sciendo.com/ & All fields \\
\hline 156. & Walter de Gruyter & https://www.degruyter.com/ & All fields \\
\hline 157. & Crossref & https://search.crossref.org/ & All fields \\
\hline 158. & John Hopskin University & https://www.press.jhu.edu/journals/ & All fields \\
\hline 159. & Scholars Portal Journal & https://journals.scholarsportal.info/ & All fields \\
\hline
\end{tabular}

